\title{
The SAAP pipeline and database: tools to analyze the impact and predict the pathogenicity of mutations
}

\author{
Nouf S Al-Numair, Andrew CR Martin
}

From SNP-SIG 2012: Identification and annotation of SNPs in the context of structure, function, and disease Long Beach, CA, USA. 14 May 2012

\begin{abstract}
Background: Understanding and predicting the effects of mutations on protein structure and phenotype is an increasingly important area. Genes for many genetically linked diseases are now routinely sequenced in the clinic. Previously we focused on understanding the structural effects of mutations, creating the SAAPdb resource.

Results: We have updated SAAPdb to include 41\% more SNPs and 36\% more PDs. Introducing a hydrophobic residue on the surface, or a hydrophilic residue in the core, no longer shows significant differences between SNPs and PDs. We have improved some of the analyses significantly enhancing the analysis of clashes and of mutations to-proline and from-glycine. A new web interface has been developed allowing users to analyze their own mutations. Finally we have developed a machine learning method which gives a cross-validated accuracy of 0.846 , considerably out-performing well known methods including SIFT and PolyPhen2 which give accuracies between 0.690 and 0.785 .
\end{abstract}

Conclusions: We have updated SAAPdb and improved its analyses, but with the increasing rate with which mutation data are generated, we have created a new analysis pipeline and web interface. Results of machine learning using the structural analysis results to predict pathogenicity considerably outperform other methods.

\section{Background}

The explosion in the availability of mutation data, resulting from the application of SNP chips [1] and next-generation sequencing [2] has led to a huge demand to analyze and predict the effects of mutations. The genes for many genetically linked diseases are now routinely sequenced in the clinic.

While a mutation is defined as 'any change in the DNA', most work has focused on studying 'Single Nucleotide Variations' (SNVs). Broadly these can be classified into Single Nucleotide Polymorphisms (SNPs) and pathogenic deviations (PDs). SNPs which, if strictly defined, occur in at least $1 \%$ of a normal population, are estimated to occur once every 100-300 bases in the human genome [3], giving

\footnotetext{
* Correspondence: andrew@bioinf.org.uk Institute of Structural and Molecular Biology, Division of Biosciences, University College London, Darwin Building, Gower Street, London WC1E 6BT, UK
}

rise to subtle phenotypic variation without causing major deleterious phenotypic changes; PDs occur at much lower frequencies and are causative of disease.

In reality, SNVs form a spectrum from completely silent SNPs at one end, to $100 \%$ penetrance, Mendelianly inherited PDs at the other end. In between, SNVs show partial penetrance; that is, only a fraction of individuals having the mutation show altered phenotype and this can be influenced by the presence of other mutations and/or environmental factors.

To date, most effort has gone into understanding the effects of missense SNVs that lead to changes in protein sequence. We use the term 'Single Amino Acid Polymorphism' (SAAP) to refer to such amino acid changes whatever the frequency and resulting phenotype of the mutation. More than a dozen groups have devised methods to analyze the effects a given SAAP will have and in some cases attempt to predict whether the mutation will 
have a deleterious effect on phenotype [4-15]. However, the best known methods are SIFT [16] (an evolutionary method which calculates a sophisticated residue conservation score from multiple alignment) and PolyPhen-2 [17] which uses machine learning on a set of eight sequenceand three structure-based features. A recent addition to the set of tools is Condel [18], a consensus predictor which makes use of SIFT, PolyPhen-2 and MutationAssessor [12]. Condel significantly outperforms any of its component predictors. Until recently, rather than trying to predict whether a given SAAP will result in a deleterious phenotype, our focus has been on trying to understand the effects that mutations have on protein structure, comparing these effects in SNPs (that is non-pathogenic mutations) and PDs. Our approach has been to map SAAPs onto protein structure and to perform a rule-based analysis of the likely structural effects of these mutations in order to 'explain' the functional effect (if any) of the mutation. Since we map mutations to structure, we only consider mutations in proteins for which a structure has been solved. Data resulting from the analysis of SNPs and PDs have been collected into a relational database and made available over the web in the resource SAAPdb [19] (http://www.bioinf.org.uk/saap/db/).

In this paper we describe (i) an update of the data in SAAPdb, (ii) enhancements to methods used to analyze the structural impact of SNPs, (iii) a new web interface allowing the analysis of new mutations and (iv) results of the application of machine learning to predict the phenotypic effects of mutations based on our structural analyses.

\section{Results and discussion \\ SAAPdb update}

Considerable effort has been made to improve the code for updating SAAPdb. A summary of the datasets comparing the old and new builds of the database is shown in Table 1, while Figure 1 (which can be compared with the Hurst et al. paper [19]) shows a comparison of structural effects seen for SNPs and PDs. Other sources of mutation data have been considered including HGMD and SwissProt Variants (SwissVar). However HGMD data are only available to registered users meaning that we have not been able to reproduce their data in our database and SwissVar is not terribly reliable in annotation of disease status. For example, known PDs in G6PD are annotated as 'Natural Variants' of Unclassified disease status. Other locus specific mutation databases (LSMBDs) can easily be added [20], but as explained below, we have now implemented SAAPdap, a pipeline version of the system allowing users to analyze novel mutations, which we now regard as our primary resource.

Since we map mutations to protein structure and therefore require a structure to be solved of the protein

Table 1 Number of distinct mutations from different sources that have been mapped to protein sequence and included in SAAPdb

\begin{tabular}{|c|c|c|}
\hline Data source & Previous build & Current build \\
\hline \multicolumn{3}{|l|}{ SNPs } \\
\hline dbSNP & 34342 & 48452 \\
\hline \multicolumn{3}{|l|}{ PDs } \\
\hline OMIM & 7249 & 9339 \\
\hline ADABase Adenosine DeAminase deficiency & 30 & 38 \\
\hline $\begin{array}{l}\text { Hamsters The Haemophilia A Mutation, Structure, Test } \\
\text { and Resource }\end{array}$ & 530 & 628 \\
\hline $\begin{array}{l}\text { IARC-p53-Germline Tumor Protein } 53 \text { gene germline } \\
\text { mutation in familial cancers }\end{array}$ & 95 & 138 \\
\hline $\begin{array}{l}\text { IARC-p53-Somatic Tumor Protein } 53 \text { gene somatic mu- } \\
\text { tations in sporadic cancers }\end{array}$ & 617 & 1368 \\
\hline G6PD Glucose-6-Phosphate Dehydrogenase & 170 & 170 \\
\hline OTC Ornithine TransCarbamylase (OTC) & 148 & 217 \\
\hline SODdb SuperOxide Dismutase 1 & 96 & 125 \\
\hline ZAP70Base Zeta-chain-Associated Protein kinase 70 & 5 & 5 \\
\hline Kinbase Somatic protein kinase driver mutations & 66 & 66 \\
\hline Kinbase Somatic protein kinase passenger mutations & 66 & 66 \\
\hline LDLR Low Density Lipoprotein Receptor & 516 & 515 \\
\hline PAHdb Human Phenylalanine Hydroxylase gene & 0 & 337 \\
\hline STAT3 Signal Transducer and Activator of Transcription 3 & 0 & 47 \\
\hline Total PDs & 9588 & 13059 \\
\hline
\end{tabular}




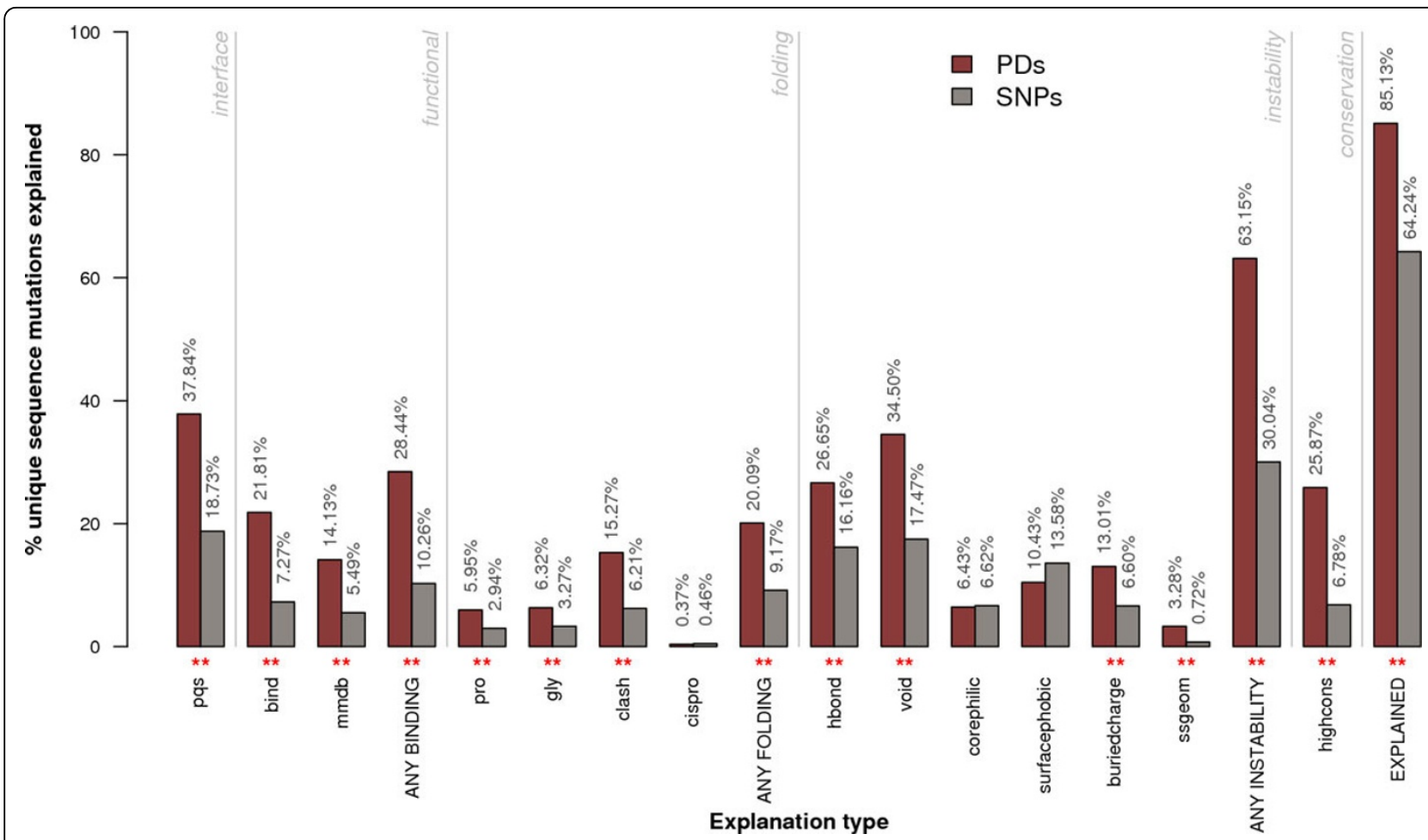

Figure 1 PQS Residue in a contact with a different protein chain or ligand, according to the PQS server; Bind Residue in a contact with a different protein chain or ligand, according to PDB data; MMDB Residue in a contact with a ligand, according to the MMDB server; Gly Mutation from glycine, introducing unfavourable torsion angles; Pro Mutation to proline, introducing unfavourable torsion angles; Cispro Mutation from cisproline, introducing unfavourable torsion angle; Clash Mutation introducing a steric clash with an existing residue; Void Mutation introducing a destabilizing void $>275 \AA^{3}$ in the protein core; Hbond Mutation disrupting a hydrogen bond; CorePhilic Introduction of a hydrophilic residue in the protein core; SurfacePhobic Introduction of a hydrophobic residue on the protein surface; BuriedCharge Mutation causing an unsatisfied charge in the protein core; SSgeom Mutation disrupting a disulphide bond; HighCons Residue has conserved sequence; EXPLAINED Any of the above categories. In addition, we look at whether a residue annotated as functionally relevant by UniProtKB/SwissProt; Asterisks indicate a significant result (two where $p<0.01$ and one where $p<0.05)$ calculated as described in Hurst et al. [19].

of interest, we are not able to analyze all mutations. Of the amino acid mutations in OMIM, we are only able to map approximately $57 \%$ to structure, while only approximately $22 \%$ of 'valid' SNPs from dbSNP, which result in an amino acid change, map to structure. Consequently the coverage of our analysis is currently somewhat limited, but clinically relevant proteins tend to be key targets for structural studies, so we expect this figure to improve. Where multiple structures have been solved, we analyze the effects of the mutations in all available structures.

In summary, the number of SNPs in the database has risen by $41 \%$ and the number of PDs by $36 \%$ (including two new sources of mutation data). The comparison of structural effects between SNPs and PDs shows the same trends as in the previous analysis, but the 'surfacephobic' (introducing a hydrophobic residue onto the surface) and 'corephilic' (introducing a hydrophilic residue into the core) analyses no longer show significant differences between SNPs and PDs.

\section{Analysis enhancements}

In SAAPdb, all assignments of structural effects are Boolean - that is, any mutation either does, or does not, have a given effect. While Boolean assignment is appropriate in some cases (for example, a residue either is, or is not, annotated as a feature in UniProtKB/SwissProt), in other cases, it relies on some cutoff (for example, energy, void volume, hydrophobicity difference) as described previously [19,21-23].

We found that assigning a mutation as (not) having a structural effect is very sensitive to precise structural details; where multiple structures are available for the same protein, one structure may indicate that a mutation has a value just below a cutoff while another structure has a value just above. Wherever appropriate, we have now implemented real-number scores or pseudoenergies for each effect. In particular, we have enhanced the analysis of clashes and torsion angles to provide energy values. 


\section{Clashes}

In analyzing clashes, our previous work defined a damaging clash as any sidechain that has at least 3 van der Waals overlaps (of any degree) with other atoms [19]. We now perform a more complete energy calculation incorporating Lennard-Jones and torsion energies using CHARMM [24] parameters:

$$
E=\left(\frac{A}{r^{12}}-\frac{B}{r^{6}}\right)+k(1+\cos (n \psi+\phi))
$$

This accounts for any clashes between atoms of the sidechain being replaced with its surroundings, together with preferences for staggered conformations (see Figure 2). Testing the new method on 400,000 residues from CATH O-representatives (domains having no more than $65 \%$ sequence identity) of high resolution $(<2.5 \AA)$ shows that $99 \%$ of sidechains have an energy of $<13.4 \mathrm{kcal} / \mathrm{mol}$ (see Figure 3).

Using the new energy evaluation we went on to analyze how the old clash method performed. In the old method, no account was made of the degree of clash - overlaps of $0.01 \AA$ or of $1.0 \AA$ were treated the same. Figure 4 shows the energy distribution for sidechain replacements considered to make 0-5 clashes by the old method. Looking at sidechain replacements that made no clashes using the old method (Figure 4, panel 1), we see that $99 \%$ of the data have an energy below $34.33 \mathrm{kcal} / \mathrm{mol}$ using the new energy-based method. Panels 2 and 3 show cases evaluated as making one or two clashes using the old method.

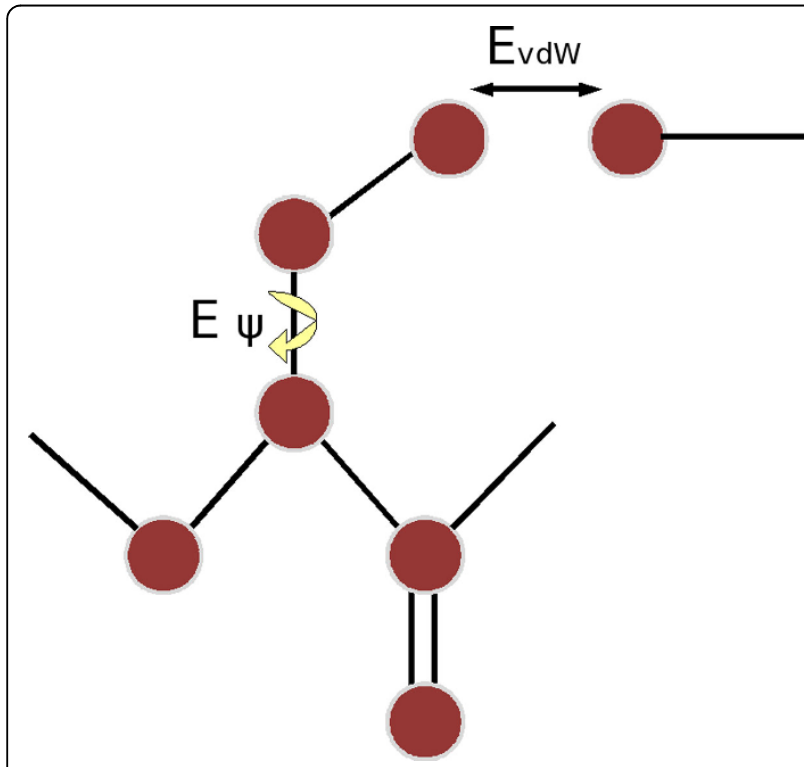

Figure 2 Schematic indicating the two new terms used in evaluation of clashes. $E_{v a w}$ is the van der Waals energy evaluated using a standard Lennard-Jones potential while $E_{\psi}$ is a torsion energy.

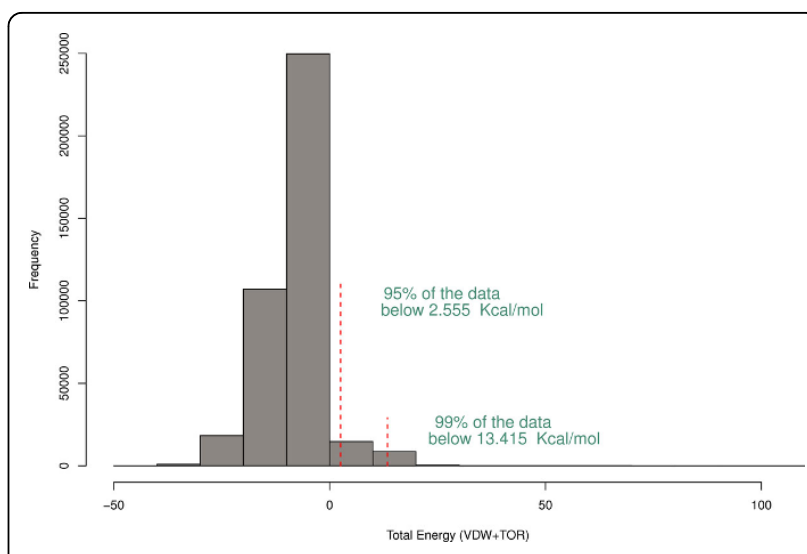

Figure 3 Distribution of sidechain clash energies calculated according to Equation 1 for high resolution structures amongst CATH O-representatives

Using $34.33 \mathrm{kcal} / \mathrm{mol}$ as an energy cutoff, these graphs indicate that $33.2 \%$ and $28.9 \%$ of potentially damaging clashes (shaded regions in panels 2 and 3 respectively) were not detected using the old method. Panels 4, 5 and 6 show the energy distributions for sidechain replacements having 3-5 clashes by the old method which would have been classified as damaging. However, using the new method, $19.5 \%, 10.7 \%$ and $11.2 \%$ of cases (shaded regions in panels 4, 5 and 6 respectively) have energies below the threshold and are therefore unlikely to be damaging.

Overall, approximately $32 \%$ of mutations previously classified as not clashing are now found to clash while approximately $15 \%$ of mutations previously classified as clashing are now found to have only minor clashes which could be relieved by very slight movements in the structure.

\section{Glycine and proline mutations}

Glycine and proline are the 'structural' amino acids which show an unusual Ramachandran distribution. Because glycine has no sidechain, it is able to access a wider range of phi/psi combinations while the cyclic sidechain of proline restricts the available phi angles. Consequently, backbone conformational changes may be necessary to accommodate mutations from-glycine or to-proline.

Previously, we used a very simple set of allowed boundaries for backbone phi/psi angles. We have now developed a pseudo-energy potential based on Ramachandran plots. A non-redundant set (sequence identity $<25 \%$ ) of high resolution protein domains (resolution $\leq 1.8 \AA$, R-Factor $\leq 0.3$ ) was selected from CATH and Ramachandran plots were generated on a 1-degree grid for proline, glycine and 'other' amino acids. The plot was smoothed by averaging each of the cells with its eight neighbours (Figure 5). For each cell, we can then calculate: 


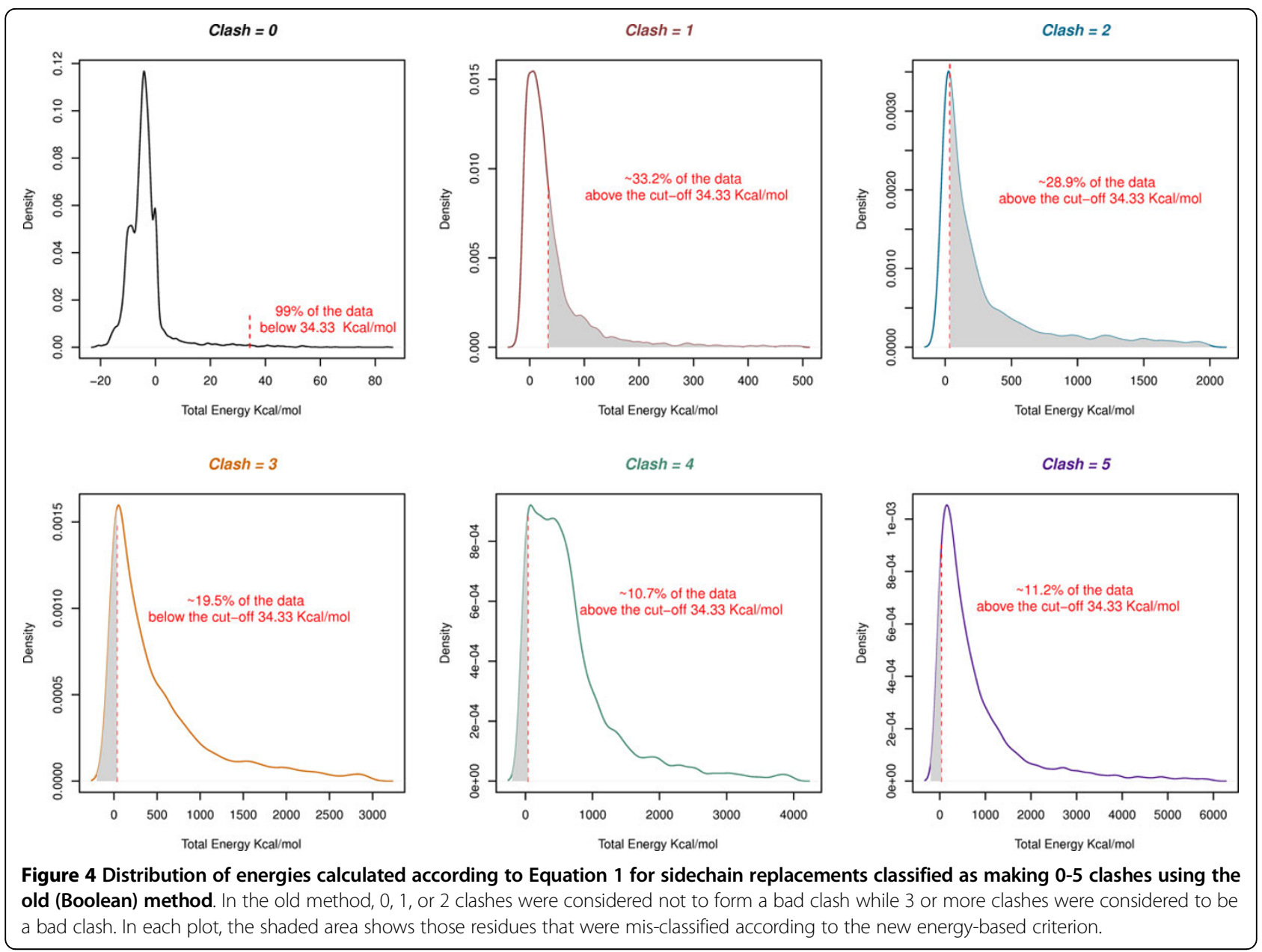

$$
E=-\log \left(\frac{\mathrm{obs}}{\exp }\right)
$$

where 'obs' is the (smoothed) observed number of residues with a given phi/psi combination while 'exp' is the expected number, calculated as the total number of observations divided by the number of cells. A threshold energy was calculated for each plot based on $1 \%$ of observations in high quality non-redundant structures having a worse energy.

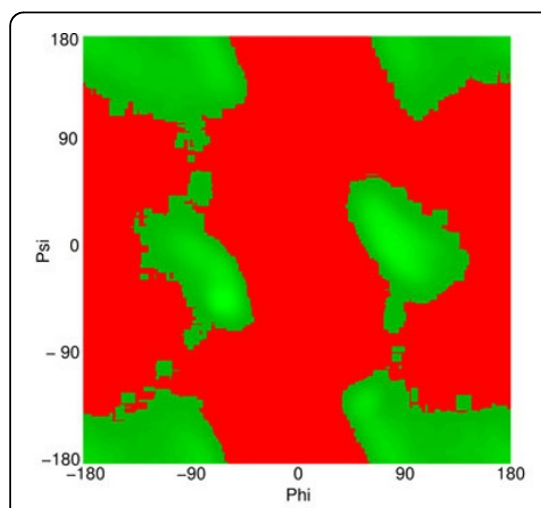

a)

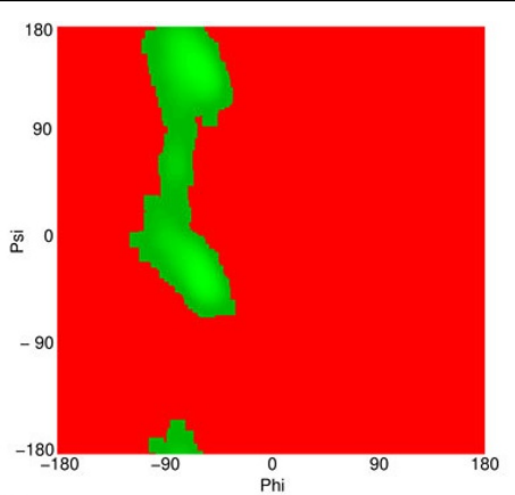

b)

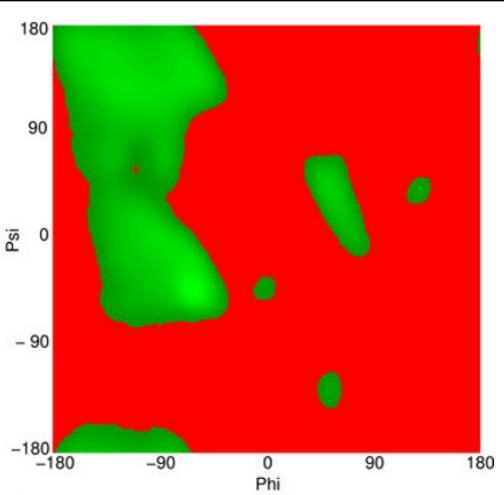

c)

Figure 5 Ramachandran plots generated from high resolution structures. a) glycine, b) pro-line, c) other. Favoured regions are shown in progressively paler green while disfavoured regions are shown in red. 


\section{SAAP data analysis pipeline (SAAPdap)}

SAAPdb was designed as a regularly updated pre-calculated resource. However, it has proved very difficult to maintain and changes in licensing of OMIM data mean that we may no longer be able to use this as our primary source of PDs. In addition, with the increasing routine use of high-throughput sequencing methods to detect mutations, more and more people want to be able to analyze their own mutations.

Consequently we believe the value of SAAPdb has diminished and have now implemented SAAPdap (Single Amino Acid Polymorphism Data Analysis Pipeline). This is a complete rewrite of the mutation analysis software in SAAPdb using a plugin architecture and making use of the new non-Boolean analyses. While we still indicate whether a mutation is likely to have a detrimental effect on structure using cutoff values, we also provide continuous values for each of the analyses.

Results from the SAAPdap pipeline are presented as shown in Figure 6a. Results are summarized at the top where the effects on each structure to which the mutation maps are shown. Below, the analyses of structural effects on each structure are presented and these can then be expanded to provide more detail on the analyses as shown in Figure 6b. Analysis descriptions are much more comprehensive than was the case in SAAPdb to make the results easier to understand.

We have now implemented a web interface to allow users to enter mutations for analysis. Because some of the analyses (especially the analysis of voids) are quite time consuming (taking several minutes), the web interface makes use of AJAX (Asynchronous JavaScript And XML) to update the user with the progress of the analysis. The submission page is available at http://www.bioinf.org.uk/ saap/dap/.

\section{Predicting damaging mutations (SAAPpred)}

The data in SAAPdb (Figure 1) show clear differences in the sequence and structural characteristics of SNPs and PDs: PDs have additional, and more severe, structural effects. Thus there is a clear signal that can be used to predict the pathogenicity of a novel mutation.

In preliminary experiments, we used a balanced set of mutation data from SAAPdb with Random Forests implemented in Weka obtaining an accuracy (Acc) of 0.935 and a Matthews Correlation Coefficient (MCC) of 0.871 (based on 10-fold cross-validation). The balanced dataset consisted of 30,500 SNPs mapped to unique PDB structures (see Table 2) and processed without any errors, and a random selection of 30,500 PDs (mapped to unique PDBs). Where several structures are available for a mutated residue, each is used as an independent data point in training the machine learning. While the 10-fold cross-validation in Weka ensures that there is no direct overlap between the training and test sets, in these preliminary experiments, there may be some 'structural' overlap - in other words, for a given mutation in the test set, the same mutation in a different PDB file of the same protein may be present in the training set.

González-Pérez and López-Bigas [18], report that well known individual methods (SIFT, PolyPhen2, Logre [25], MAPP [26] and MutationAssessor) give accuracies between 0.690 and 0.771 evaluated on the HumVar dataset developed for PolyPhen2. Their consensus method (Condel) gives an accuracy of 0.882 . While our preliminary value of 0.935 is considerably better, we are using a different dataset.

However, having trained on SAAPdb, if we test on 1540 SNPs and 7182 PDs from the HumVar dataset that mapped to structure we obtain Acc $=0.446, \mathrm{MCC}=0.135$ -essentially a random prediction. This appears to be because of the different definition of the 'boundary' between SNPs and PDs. As stated above, SNVs form a spectrum from completely silent SNPs at one end, to $100 \%$ penetrance, Mendelianly inherited PDs at the other end. As shown in Figure 7, different datasets use different thresholds to separate the data into two sets or may consider only the extremes. Prediction of the extremes may appear to be a trivial problem, but this is not always the case - some damaging mutations are very hard to predict. HumVar uses a broader definition of PDs than the SAAPdb data; in contrast, the SAAPdb definition of SNPs is rather wide (anything in dbSNP not annotated as being involved in disease) while the definition in HumVar enforces the requirement that SNPs are present in at least $1 \%$ of a normal population. Since, in this experiment, we use very different definitions for the training and testing, it is not surprising that we obtain poor performance. We considerably over-predict SNPs, consistent with our broader definition of SNPs in the SAAPdb dataset.

Consequently, for the final version of SAAPpred, we both trained and tested our method on the HumVar dataset (using 10-fold cross-validation). HumVar (2011/ 12) contains 22,196 deleterious mutations and 21,151 neutral mutations of which 7,182 and 1,540, respectively, can be mapped to structure. Consequently, to obtain a balanced dataset, only 3,080 mutations (equal numbers of deleterious and neutral) can be used. Ten runs were performed, each of which used all 1,540 neutral mutations with a random selection of 1,540 deleterious mutations from the total of 7,182. Results from the ten runs were then averaged. In each run, to avoid the 'structural overlap' between the training and testing data during cross-validation (which was present in the preliminary experiments with SAAPdb data), the mutations were split into 10 sets of approximately the same size. Each 
a)

SAAP Analysis

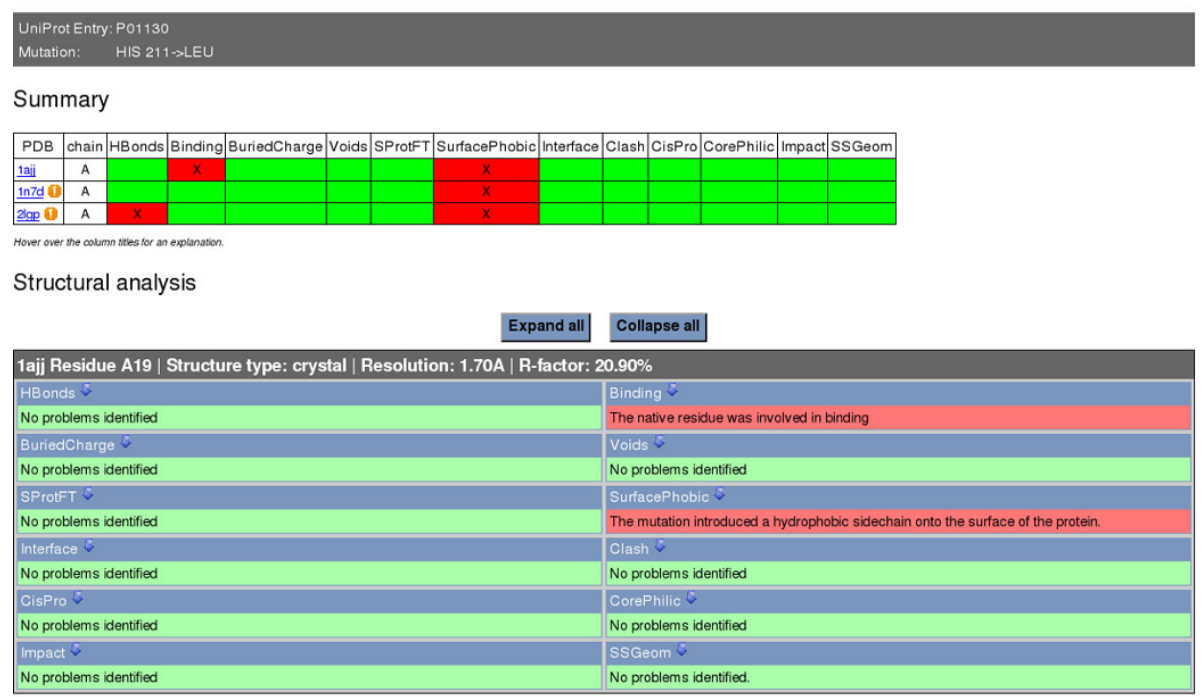

b)

\begin{tabular}{|c|c|}
\hline HBonds $\hat{?}$ & Binding $\hat{?}$ \\
\hline $\begin{array}{l}\text { No problems identified } \\
\text { The native residue was not involved in a hydrogen bond }\end{array}$ & $\begin{array}{l}\text { The native residue was involved in binding } \\
\text { A specific HBond or van der Waals interaction occurred with another protein or ligand }\end{array}$ \\
\hline BuriedCharge $\hat{\imath}$ & Voids $\hat{x}$ \\
\hline $\begin{array}{l}\text { No problems identified } \\
\text { Native residue charge: } 1 \\
\text { Mutant residue charge: } 0 \\
\text { Relative accessibility of native residue: } 58.112 \%\end{array}$ & $\begin{array}{l}\text { No problems identified } \\
\text { After the mutation the largest void was of size } 17.68 \text {. } \\
\text { The largest void in the native was } 17.68 \text {. } \\
90 \% \text { of proteins have no volds s } 275, \text { but we do not consider a large void to be damaging if the } \\
\text { native structure had large voids. }\end{array}$ \\
\hline SProtfT $\hat{3}$ & Surface Phobic $\hat{\text { ? }}$ \\
\hline $\begin{array}{l}\text { No problems identified } \\
\text { The residue was not annotated as a feature' in UniP rotKB/SwissProt }\end{array}$ & $\begin{array}{l}\text { The mutation introduced a hydrophobic sidechain onto the surface of the protein. } \\
\text { Native residie hydrophobicity: - } 0.4 \\
\text { Mutant residue hydrophobicity } 0.53 \\
\text { Relative accessiblity of native residue: } 58.112 \% \\
\text { Hydrophobicity values }<0 \text { are hydrophilic and a threshold of } 20 \% \text { relative accessibility is used } \\
\text { to define a residue as on the surface. }\end{array}$ \\
\hline Interface $\hat{?}$ & Clash ? \\
\hline $\begin{array}{l}\text { No problems identified } \\
\text { Interfaces are defined by a difference in solvent accessibility between a complex and the } \\
\text { individual chain in the crystal structure. } \\
\text { In the complex, this residue had a relative accessability of } 48.599 \% \text { while the individual chain } \\
\text { had an accessibility of } 58.112 \% \text { a diffterence of } 9.513 \% \\
\text { A difference of }>10 \% \text { is taken as indicative of an interface residue. } \\
\text { Interfaces may be with another protein chain or a ligand. }\end{array}$ & $\begin{array}{l}\text { No problems identified } \\
\text { The clash energy was }-8.75 \mathrm{kcal} / \mathrm{mol} \text {. } \\
99 \% \text { of sidechains in real proteins have an energy less than } 34.33 \mathrm{kcal} / \mathrm{mol} \text {. } \\
\text { Consequently energies }>34.33 \mathrm{kcal} / \mathrm{mol} \text { and }<50 \mathrm{kccal} \text { mol can be considered mild clashes, } \\
50-100 \mathrm{kca} / \mathrm{mol} \text { medium clashes, }>100 \mathrm{kca} / \mathrm{mol} \text { severe clashes. Note that clash energies } \\
\text { can be extremely high ( }>>100000 \mathrm{kcal} / \mathrm{mol})\end{array}$ \\
\hline CisPro $A$ & CorePhilic $\hat{\imath}$ \\
\hline $\begin{array}{l}\text { No problems identified } \\
\text { The native residue was not a cis-proline }\end{array}$ & $\begin{array}{l}\text { No problems identified } \\
\text { Native residue hydrophobicity: }-0.4 \\
\text { Mutant residue hydrophobicity: } 0.53 \\
\text { Relative accessibility of native residue: } 58.112 \% \\
\text { Hydrophobicity values }<0 \text { are hydrophilic. }\end{array}$ \\
\hline Impact $\hat{\jmath}$ & SsGeom र̂ \\
\hline $\begin{array}{l}\text { No problems identified } \\
\text { In an alignment of } 5 \text { functionally equivalent protein sequences, the conservation score at } \\
\text { this position was } 31 \% \text {. } \\
\text { Analysis of the sequences in this alignment defined a threshold of } 84 \% \text { as being significantly } \\
\text { conserved. } \\
\text { Note that since there are < } 10 \text { sequences in the alignment, this result should be treated with } \\
\text { caution! }\end{array}$ & $\begin{array}{l}\text { No problems identified. } \\
\text { The native residue was not a cysteine in a disulphide bond. }\end{array}$ \\
\hline
\end{tabular}

Figure 6 Results pages from the new SAAPdap pipeline. a) Summary and brief structural reports - hovering over any of the titles brings up a box to explain the meaning of the effect; b) Expanded view of full structural analysis.

of these 10 sets in turn was chosen as a test set. The remaining 9 sets were used for training by randomly drawing balanced datasets of different sizes from the mutations as mapped to protein chains (see Table 2). This manual cross-validation ensures that there are no cases of the same mutation in the training and test sets but from different PDB chains.
As expected, the performance $(\mathrm{Acc}=0.846, \mathrm{MCC}=$ 0.692) is rather worse than with the SAAPdb data, simply because the size of the HumVar dataset that can be mapped to structure is much smaller than the SAAPdb dataset. Figure 8 shows the effect of dataset size on training and testing using subsets of the SAAPdb data. The same procedure described above was used to avoid 
Table 2 Breakdown of the number of mutations in SAAPdb and their mapping to structure.

\begin{tabular}{lll}
\hline Number of Mutations & PDs & SNPs \\
\hline Mapped to UniProtKB/SwissProt & 13,059 & 48,452 \\
Mapped to PDB & 6,527 & 17,915 \\
Mapped to multiple PDBs & 202,566 & 33,369 \\
Mapped to multiple Chains & 405,497 & 45,699 \\
\hline
\end{tabular}

In some cases, several hundred structures are available (e.g. haemoglobin, carbonic anhydrase, prthrombin, transthyretin, insulin, CDK2, lysozyme) and, on average there are approximately two copies of each chain in each PDB file.

structural overlap between training and testing sets during cross-validation. The graph clearly shows that the smaller datasets perform considerably worse.

Nonetheless, our results from training and testing on HumVar mutations that map to structure considerably outperform other well-known individual methods including SIFT and PolyPhen2 as reported by González-Pérez and López-Bigas [18] (Accuracies between 0.690 and 0.771 ). However these results are still not directly comparable with the other methods as those methods are evaluated on the complete HumVar dataset and it may be argued that the subset of mutations for which structures are available somehow outperform those for which structures are not available in these other methods. For example, PolyPhen 2 makes limited use of structural data where these are available.

Consequently, we have evaluated the performance of PolyPhen2, SIFT and MutationAssessor using balanced datasets (1451 neutral mutations and ten random selections of 1451 deleterious mutations) used to assess the performance of our method. (Note that we could only use 1451 rather than 1540 mutations since the remaining 89 PDs failed in at least one of the other predictors.) In fact this gives a significant advantage to PolyPhen2 which is itself trained on HumVar leading to an overlap between the training and test set. It is not clear precisely what data are used to train SIFT; in their latest paper, Sim et al. [27]. state that SIFT was originally trained and tested on LacI, Lysozyme and HIV protease, and refer to the original SIFT papers, but they do not state whether the training has since been modified. MutationAssessor does not appear to use a training set per se.

Results are summarized in Table 3 where it can be seen that our method (SAAPpred) evaluated using 10-fold cross-validation (i.e. with no overlap between test and training sets) performs better than competing methods where there may be overlap between testing and training data. In particular, PolyPhen-2 was trained on the complete HumVar dataset from which our test set is extracted. If we allow overlap in our own set (the fairest comparison) then we outperform PolyPhen2 (the best of the competing methods) by an even larger margin.

While SAAPpred is clearly performing extremely well, we expect to be able to improve results further through feature selection (to help with the relatively small HumVar dataset size), feature combination (e.g. subtracting native void sizes from mutant void sizes) and feature normalization (e.g. taking the log of some feature values to improve the distribution of values). We also hope to develop methods to make more complete use of unbalanced datasets and intend to use our predictor as a component predictor of the consensus predictor Condel [18] which outperforms the other individual methods $($ Acc $=0.882)$.

\section{Conclusions}

In conclusion, we have updated the data in SAAPdb, improved the analyses and integrated these into the new SAAPdap pipeline and web interface. It is intended that SAAPdap will replace SAAPdb (which has proved difficult to update regularly and reliably). The submission page for SAAPdap is available at http://www.bioinf.org.uk/saap/ dap/.

We are currently working on new analyses that examine sequence differences at the DNA and RNA level. In addition to changes to the protein structure, mutations can have effects on expression, RNA splicing and RNA folding and stability [28-30].

Results of machine learning using the structural parameters calculated in SAAPdap considerably out-perform any other individual predictor and approaching the performance of the combined predictor, Condel. Future work

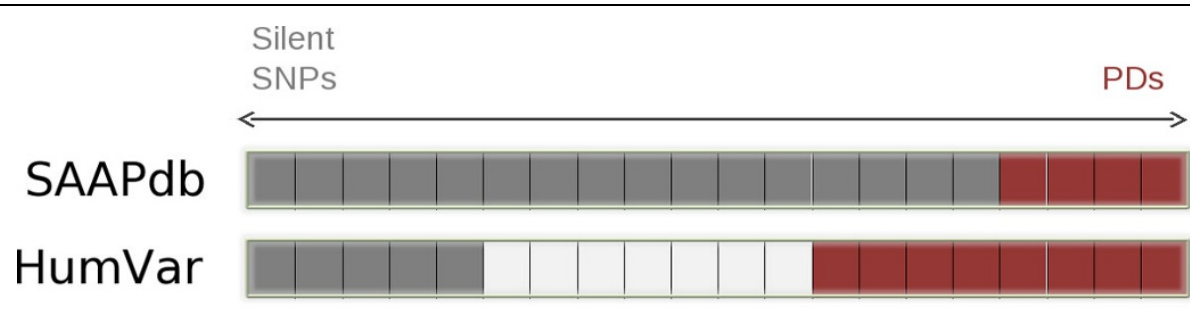

Figure 7 The penetrance of a mutation lies on a scale between 'True SNPs' which show no phenotypic effect at one extreme to Mendelianly inherited PDs with $\mathbf{1 0 0} \%$ penetrance at the other. In SAAPdb, we use a very conservative definition of PDs, but a rather wide definition of SNPS. In contrast, HumVar uses a somewhat broader definition of PDs, but a much more conservative definition of SNPS and does not consider mutations that lie in the middle. 


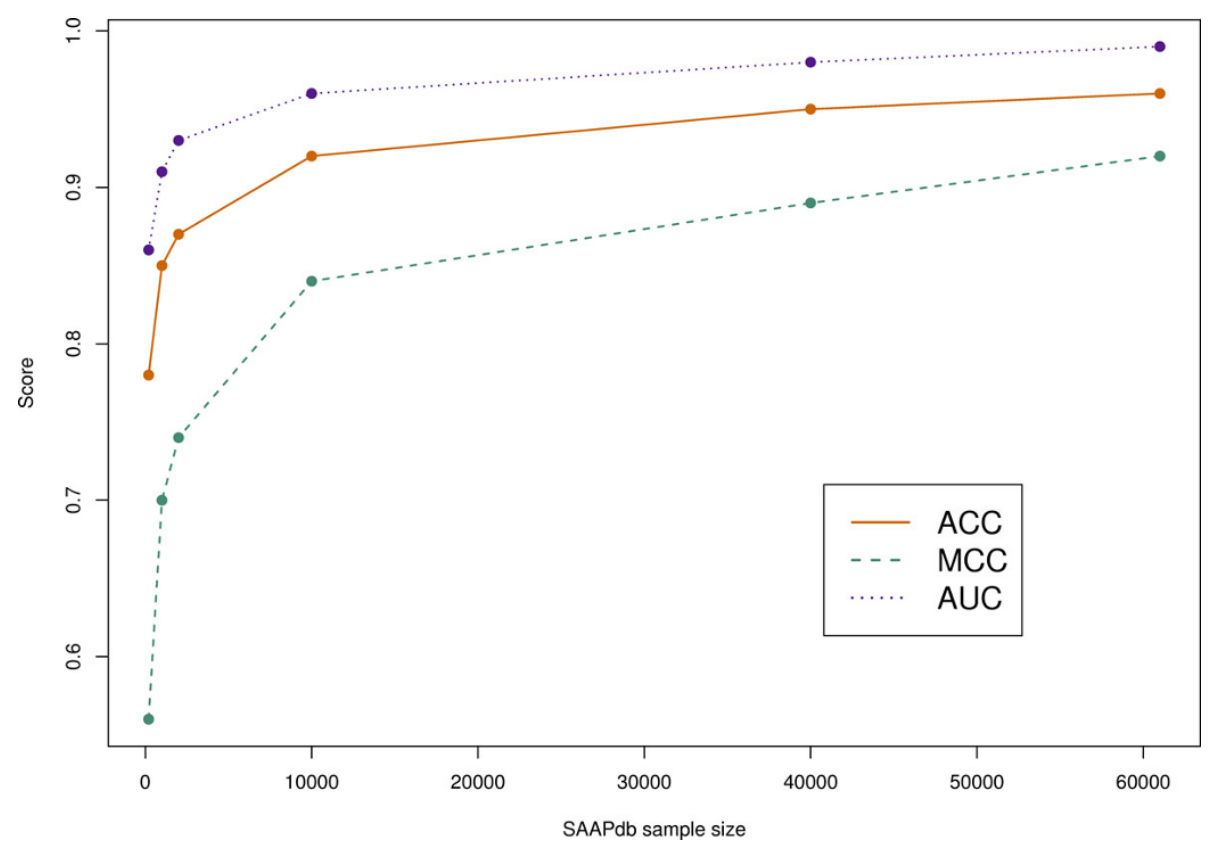

Figure 8 Performance of the machine learning method trained on different sized sets of data from SAAPdb. In each case, a balanced dataset of the required size was extracted at random from the SAAPdb dataset of mutations mapped to protein chains (Table 2) and random forests were trained and tested using 10 -fold cross-validation. The graph clearly shows that performance drops as the dataset size decreases, showing a marked drop in performance with datasets below 10,000 samples in size (5,000 SNPs and 5,000 PDs).

will further optimize the performance of this method using feature selection, feature combination and feature normalization as well as exploiting strategies to make more complete use of unbalanced datasets. We will integrate our predictor as a component of Condel and expect performance to outperform the current Condel method.

While the coverage of our method is currently somewhat limited by the need for a structure of the protein, we

Table 3 Performance of different prediction methods using a balanced dataset of mutations that map to structure extracted from HumVar.

\begin{tabular}{llll}
\hline Method & Cross-validated & MCC & Acc \\
\hline SAAPpred & Yes & 0.692 & 0.846 \\
SAAPpred & No & 0.894 & 0.944 \\
PolyPhen2 & No & 0.572 & 0.785 \\
SIFT & $?$ & 0.528 & 0.763 \\
MutationAssessor & N/A & 0.453 & 0.698 \\
\hline
\end{tabular}

The values for the cross-validated assessment of SAAPpred were obtained from 10-fold cross-validation performed during the Weka training and used all 1540 SNPs from HumVar that mapped to structure with a random sample of 1540 of the 7182 PDs that mapped to structure. This was repeated 10-times and the results averaged. Non cross-validated results were performed by using a slightly smaller set of 1451 SNPs that mapped to structure and could be assessed by all the other methods together with a random sample of 1451 PDs that could be assessed by all methods. Again this was repeated 10-times and the results averaged. The non-cross-validated values for SAAPpred give the fairest comparison with PolyPhen2 which is trained on the HumVar dataset. It is unclear exactly what data were used in training the most recent version of SIFT so there may be some overlap between training and test sets while MutationAssessor has no training set per se. plan to investigate the use of modelled structures. However, we currently don't know how well this will work given the detailed structural analysis (e.g. of hydrogen bonds) that our method performs. However clinically relevant proteins tend to be key targets for structural studies, and as more structures become available, the number of mutants mapped to structure will increase, improving the coverage of our method. In addition, more structural data will allow us to train the machine learning methods with more data. Consequently, as shown in Figure 8, we expect performance to increase further.

\section{Materials and methods}

SNP data were extracted from the XML format dump of dbSNP [31] obtained from the NCBI. Non-synonymous, 'valid' human SNPs (i.e. those annotated with validation strings 'by frequency', 'by 2hit 2allele', or 'by hapmap'), were extracted and combined into a single XML file. Any mutations not annotated as having disease involvement were assumed to be neutral. PDs were obtained from Online Mendelian Inheritance in Man (OMIM, http://www.ncbi.nlm.nih.gov/omim/) and a number of locus-specific mutation databases ('LSMDBs') [20], see Table 1. All mutations were then mapped to protein sequences and thence to structure as described previously [19].

SAAPdb and SAAPdap perform fourteen analyses: Interface: (or PQS:) Residue is in an interface according 
to solvent accessibility criteria; Binding: Residue makes specific interactions with a different protein chain or ligand; SprotFT: Residue is annotated as functionally relevant by UniProtKB/SwissProt; Clash: Mutation introduces a steric clash with an existing residue; Void: Mutation introduces a destabilizing void in the protein core; Cis-Proline: Mutation from cis-proline, introducing an unfavorable omega torsion angle; Glycine: Mutation from glycine, introducing unfavorable torsion angles; Proline: Mutation to proline, introducing unfavorable torsion angles; HBond: Mutation disrupts a hydrogen bond; Corephilic: Introduction of a hydrophilic residue in the protein core; Surfacephobic: Introduction of a hydrophobic residue on the protein surface; Buriedcharge: Mutation causes an unsatisfied charge in the protein core; SSgeometry: Mutation disrupts a disulphide bond; Impact: Residue is significantly conserved. From these analyses (using software written in Perl and C) we derive 47 features that are used for machine learning. Random Forests (implemented in Weka [32]) were used for all predictions. Random Forests are ensemble classifiers that consist of multiple decision trees, each of which uses a random subset of the available features. The output of the predictor is the fraction of trees voting for the most popular class (in this case PD or SNP). Initial trials were performed using SAAPdb and HumVar data with 1000 trees and between 4 and 45 features per tree. 40 features performed best with SAAPdb while 4 features performed best with HumVar and these values were used for building the final machine learning models. Data are stored in a PostgreSQL relational database.

\section{Note added in proof}

An additional predictor FATHMM (http://fathmm.biocompute.org.uk/) has become available since this work was completed (Shihab HA, Gough J, Cooper DN, Stenson PD, Barker GLA, Edwards KJ, Day INM, Gaunt, TR. (2013). Predicting the Functional, Molecular and Phenotypic Consequences of Amino Acid Substitutions using Hidden Markov Models. Hum. Mutat., 34:57-65). Evaluation of FATHMM on the same dataset shows a performance of $\mathrm{ACC}=0.836$, $\mathrm{MCC}=0.671$. While approaching our cross-validated performance, it is likely that some of the HumVar data were included in training FATHMM.

\footnotetext{
Authors' contributions

NSAN updated the SAAPdb code and data, wrote some of the enhanced analysis methods, performed the machine learning and all testing, drafted the paper and created the figures. ACRM implemented SAAPdap and the remaining enhanced analysis methods, revised the paper and contributed to the machine learning.
}

Competing interests

The authors declare that they have no competing interests.

\section{Acknowledgements}

NSAN thanks the King Faisal Specialist Hospital and Research Centre and the Royal Embassy of Saudi Arabia Cultural Bureau (reference S12063) for funding. ACRM thanks Prof Giuliano Armano, DIEE, University of Cagliari for useful discussions on machine learning.

\section{Declarations}

The publication costs for this article were funded by the above grant. This article has been published as part of BMC Genomics Volume 14 Supplement 3, 2013: SNP-SIG 2012: Identification and annotation of SNPs in the context of structure, function, and disease. The full contents of the supplement are available online at http://www.biomedcentral.com/ bmcgenomics/supplements/14/\$3

Published: 28 May 2013

\section{References}

1. Carr SM, Marshall HD, Duggan AT, Flynn SMC, Johnstone KA, Pope AM, Wilkerson CD: Phylogeographic Genomics of Mitochondrial DNA: Highlyresolved Patterns of Intraspecific Evolution and a Multi-species, Microarray-based DNA Sequencing Strategy for Biodiversity Studies. Comp Biochem Physiol Part D Genomics Proteomics 2008, 3:1-11.

2. Bentley DR: Whole-genome Re-sequencing. Curr Opin Genet Dev 2006, 16:545-552.

3. Wang P, Dai M, Xuan W, McEachin RC, Jackson AU, Scott LJ, Athey B, Watson SJ, Meng F: SNP Function Portal: a web Database for Exploring the Function Implication of SNP Alleles. Bioinformatics 2006, 22:e523-e529.

4. Yue P, Melamud E, Moult J: SNPs3D: Candidate gene and SNP Selection for Association Studies. BMC Bioinformatics 2006, 7:166-166.

5. Uzun A, Leslin CM, Abyzov A, Ilyin V: Structure SNP (StSNP): a web Server for Mapping and Modeling nsSNPs on Protein Structures with Linkage to Metabolic Pathways. Nucleic Acids Res 2007, 35:W384-W392.

6. Yip YL, Scheib H, Diemand AV, Gattiker A, Famiglietti LM, Gasteiger E, Bairoch A: The Swiss-Prot Variant page and the ModSNP Database: a Resource for Sequence and Structure Information on Human Protein Variants. Hum Mutat 2004, 23:464-470.

7. Dantzer J, Moad C, Heiland R, Mooney S: MutDB Services: Interactive Structural Analysis of Mutation data. Nucleic Acids Res 2005, 33:W311-W314.

8. Karchin R, Diekhans M, Kelly L, Thomas DJ, Pieper U, Eswar N, Haussler D, Sali A: LS-SNP: Large-scale Annotation of Coding Non-synonymous SNPs Based on Multiple Information Sources. Bioinformatics 2005, 21:2814-2820.

9. Stitziel NO, Binkowski TA, Tseng YY, Kasif S, Liang J: topoSNP: a Topographic Database of Non-synonymous Single Nucleotide Polymorphisms with and Without Known Disease Association. Nucleic Acids Res 2004, 32:D520-D522.

10. Reumers J, Schymkowitz J, Ferkinghoff-Borg J, Stricher F, Serrano L, Rousseau F: SNPeffect: a Database Mapping Molecular Phenotypic Effects of Human Non-synonymous Coding SNPs. Nucleic Acids Res 2005, 33: D527-D532.

11. Bao L, Zhou M, Cui Y: nsSNPAnalyzer: Identifying Disease-associated Nonsynonymous Single Nucleotide Polymorphisms. Nucleic Acids Res 2005, 33:W480-W482.

12. Reva B, Antipin $Y$, Sander C: Predicting the Functional Impact of Protein Mutations: Application to Cancer Genomics. Nucleic Acids Res 2011, 39 e118-e118.

13. Schwarz JM, Rödelsperger C, Schuelke M, Seelow D: MutationTaster Evaluates Disease-causing Potential of Sequence Alterations. Nat Methods 2010, 7:575-576.

14. Bromberg Y, Rost B: SNAP: Predict Effect of Non-synonymous Polymorphisms on Function. Nucleic Acids Res 2007, 35:3823-3835.

15. Bromberg Y, Yachdav G, Rost B: SNAP Predicts Effect of Mutations on Protein Function. Bioinformatics 2008, 24:2397-2398.

16. Ng PC, Henikoff S: SIFT: Predicting Amino acid Changes that Affect Protein Function. Nucleic Acids Res 2003, 31:3812-3814.

17. Adzhubei IA, Schmidt S, Peshkin L, Ramensky VE, Gerasimova A, Bork P, Kondrashov AS, Sunyaev SR: A Method and Server for Predicting Damaging Missense Mutations. Nat Methods 2010, 7:248-249.

18. González-Pérez A, López-Bigas N: Improving the Assessment of the Outcome of Nonsynonymous SNVs with a Consensus Deleteriousness Score, Condel. Am J Hum Genet 2011, 88:440-449. 
19. Hurst JM, MCMillan LEM, Porter CT, Allen J, Fakorede A, Martin ACR: The SAAPdb web Resource: a Large-scale Structural Analysis of Mutant Proteins. Hum Mutat 2009, 30:616-624.

20. Claustres M, Horaitis O, Vanevski M, Cotton RGH: Time for a Unified System of Mutation Description and Reporting: a Review of Locusspecific Mutation Databases. Genome Res 2002, 12:680-688.

21. Cuff AL, Janes RW, Martin ACR: Analysing the Ability to Retain Sidechain Hydrogen-bonds in Mutant Proteins. Bioinformatics 2006, 22:1464-1470.

22. Cuff AL, Martin ACR: Analysis of void Volumes in Proteins and Application to Stability of the p53 Tumour Suppressor Protein. J Mol Biol 2004, 344:1199-1209.

23. Martin ACR, Facchiano AM, Cuff AL, Hernandez-Boussard T, Olivier M, Hainaut $P$, Thornton JM: Integrating Mutation data and Structural Analysis of the TP53 Tumor-suppressor Protein. Hum Mutat 2002, 19:149-164.

24. Brooks B, Bruccoleri RE, Olafson BD, States DJ, Swaminathan S, Karplus M: CHARMM: A program for macromolecular energy, minimization, and dynamics calculations. J Comp Chem 1983, 4:187-217.

25. Clifford RJ, Edmonson MN, Nguyen C, Buetow KH: Large-scale Analysis of Non-synonymous Coding Region Single Nucleotide Polymorphisms. Bioinformatics 2004, 20:1006-1014

26. Binkley J, Karra K, Kirby A, Hosobuchi M, Stone EA, Sidow A: ProPhylER: a Curated Online Resource for Protein Function and Structure Based on Evolutionary Constraint Analyses. Genome Res 2010, 20:142-154.

27. Sim NL, Kumar P, Hu J, Henikoff S, Schneider G, Ng PC: SIFT web Server: Predicting Effects of Amino acid Substitutions on Proteins. Nucleic Acids Res 2012, 40:W452-W457.

28. Duan J, Wainwright MS, Comeron JM, Saitou N, Sanders AR, Gelernter J, Gejman PV: Synonymous Mutations in the Human Dopamine Receptor D2 (DRD2) Affect mRNA Stability and Synthesis of the Receptor. Hum Mol Genet 2003, 12:205-216.

29. Wang D, Sadée W: Searching for Polymorphisms that Affect gene Expression and mRNA Processing: Example ABCB1 (MDR1). AAPS J 2006, 8:E515-E520.

30. Halvorsen M, Martin JS, Broadaway S, Laederach A: Disease-associated Mutations that Alter the RNA Structural Ensemble. PLoS Genet 2010, 6 e1001074-e1001074.

31. Sherry ST, Ward MH, Kholodov M, Baker J, Phan L, Smigielski EM, Sirotkin K: dbSNP: the NCBI Database of Genetic Variation. Nucleic Acids Res 2001 29:308-311.

32. Witten IH, Frank E, Hall MA: Data Mining: Practical Machine Learning Tools and Techniques. third edition. Burlington, MA 01803, USA: Morgan Kaufmann; 2011.

doi:10.1186/1471-2164-14-S3-S4

Cite this article as: Al-Numair and Martin: The SAAP pipeline and database: tools to analyze the impact and predict the pathogenicity of mutations. BMC Genomics 2013 14(Suppl 3):S4.

\section{Submit your next manuscript to BioMed Central and take full advantage of:}

- Convenient online submission

- Thorough peer review

- No space constraints or color figure charges

- Immediate publication on acceptance

- Inclusion in PubMed, CAS, Scopus and Google Scholar

- Research which is freely available for redistribution

Submit your manuscript at www.biomedcentral.com/submit
Biomed Central 